\title{
One "8"-shaped scleral suture to treat rhegmatogenous retinal detachment: a refined procedure of minimal scleral buckling
}

\author{
H.Y. Min, D. Chen, Y. Chen and F.T. Dong \\ Department of Ophthalmology, Peking Union Medical College Hospital, \\ Chinese Academy of Medical Sciences \& Peking Union Medical College, \\ Beijing, China \\ Corresponding author: H.Y. Min \\ E-mail: hycden@126.com
}

Genet. Mol. Res. 13 (3): 6665-6671 (2014)

Received May 17, 2013

Accepted October 22, 2013

Published August 28, 2014

DOI http://dx.doi.org/10.4238/2014.August.28.11

\begin{abstract}
The aim of this study was to investigate the outcomes of one "8"-shaped scleral suture of minimal scleral buckling (MSB) surgery without sub-retinal drainage for rhegmatogenous retinal detachment (RRD) treatment. Thirty patients (30 eyes) with RRD were recruited. Thirty eyes with RRD were repaired by one " 8 "-shaped scleral suture of minimal buckling without subretinal drainage by one surgeon. The refined MSB procedure is described. Reattachment time and best-corrected visual acuity (BCVA) were observed. The age of the 30 patients ranged from 17 to 65 years (mean, $43.1 \pm 8.6$ years). The retinas of 19 eyes $(63.3 \%)$ reattached within $12 \mathrm{~h}$ of the operations, and those of 11 eyes (67\%) reattached within $72 \mathrm{~h}$. The average time of follow-up was $10.4 \pm 2.8$ months. BCVAs were increased in 27 eyes (90\%), whereas those of 3 eyes did not change. The mean preoperative BCVA was $0.738 \pm 0.368 \log$ minimal angle of resolution (MAR), and mean postoperative BCVA was $0.422 \pm 0.278 \operatorname{logMAR}$, and the difference was statistically significant $(\mathrm{P}<0.05)$. The sponge for buckling in only one eye exposed from the conjunctiva was taken out,
\end{abstract}


and the retina remained attached. In conclusion, an " 8 "-shaped scleral suture of MSB without sub-retinal drainage is an efficient procedure to treat selected RRD cases.

Key words: Best-corrected visual acuity; Minimal scleral buckling; Rhegmatogenous retinal detachment

\section{INTRODUCTION}

Rhegmatogenous retinal detachment (RRD) is characterized by one or more breaks or tears in the neurosensory retina with drainage of fluid into the subretinal space from the vitreous cavity. RRD is the most common cause of retinal detachment and greatly threatens visual acuity. The revolutionized success of a $50 \%$ reattachment rate was achieved by Juleus Gonin based on the theory of closing the break (Rumpf, 1976). In the 1950s, Schepens and colleagues introduced the scleral buckling (SB) technique for retinal detachment, which achieved higher anatomical and functional success (Schepens, 1951; Schepens et al., 1957). This classical and well-established procedure is widely applied for treating RRD, and the average reattachment rate reaches up to $90 \%$. However, severe complications do occur due to the wide buckle range, such as severe surgical trauma, diplopia, astigmatism, perforation of the eyeball, and infarction of the choroid, among others. Therefore, a method for decreasing the severity and high frequency of complications of SB is worth exploring.

Minimal scleral buckle (MSB) without sub-retinal drainage for RRD treatment was first developed by Lincoff and Kreissig on the basis of the Custodis methods (Lincoff and Kreissig, 1972). This method has been regarded as by far the best external-route surgery for which the key point is to buckle the breach only for RRD (Rezaei and Abrams, 2005). MSB is characterized by a simplified procedure, minimal surgical trauma, less and moderate complications, and better recovery, and has therefore been gradually accepted by surgeons.

However, it is difficult to define the minimal extent of MSBs. It may depend not only on the case itself, but also on the specific surgeon performing the procedure. During the operation, the surgeon always intentionally or unintentionally expands the buckle range in order to ensure that the hole or tears are on the scleral crest regardless of the size of the hole or tears (Kreissig et al., 1995; Schimel et al., 2012). Here, a refined MSB procedure without subretinal drainage was established, and the results of consecutively treating 30 eyes with RRD using this kind of surgery were investigated from January 2007 to September 2012 in the Peking Union Medical College Hospital.

\section{MATERIAL AND METHODS}

\section{Study design}

From January 2007 to September 2012, consecutive patients were recruited in the Peking Union Medical College Hospital who matched the following criteria: 1) simple RRD with only one hole or tear, 2) no fixed retinal folds, and proliferative vitreoretinopathy (PVR) less than $\mathrm{C} 1,3$ ) the position of the hole or tear was located within a 4 disc diameter (DD) around the equator, and 4) the size of the retinal hole or tear was less than 2 DD. Patients with 
macular holes, giant retinal tears, retinal dialysis, multiple holes or tears, or PVR over C2 were excluded.

The study was performed with approval of the Peking Union Medical College Hospital ethics committee. All patients provided informed consent before inclusion in the study and after the nature of the study had been explained. The study adhered to the tenets of the Declaration of Helsinki.

\section{Examinations}

After admission in the ward, antibiotic eye drops were administrated for 3 days before surgery. Bilateral lacrimal ducts were flushed with saline water. The accurate locations of the holes or tears were determined after dilation of the pupil with indirect ophthalmoscope (IO) and a three-mirror universal diagnostic lens. The sizes of the holes or tears were measured by DD. All of the retinal changes or lesions were illustrated.

\section{Surgical techniques}

During surgery, after retrobulbar anesthesia with lidocaine hydrochloride and bupivacaine, a "T"-shaped conjunctive incision was made only in the quadrant affected by the hole or tear. The sclera was exposed just enough to facilitate the location of the holes or tears. Not all of the rectus muscles were necessarily distracted by sutures. If needed, the inserted point of the muscle on the sclera was only distracted by teeth forceps to rotate the eyeball. If the hole or tears were located around the rectus muscle, it was the only one distracted by the suture in order to expose the scleral area for buckling. Using the IO, the corresponding scleral site of the retinal hole or tears was determined and marked with a Meilan marker pen. Scleral cryotherapy around the hole was applied. Subsequently, an "8"-shaped suture was tightly applied to fix the 7-mm silicone sponge buckle, which was counter-pointed to the center of the hole or tears with a 5-0 nylon stitch (Figures 1 and 2). The tie was tightened and turned to the lower edge of the sponge to prevent exposure and extrusion of the conjunctiva.
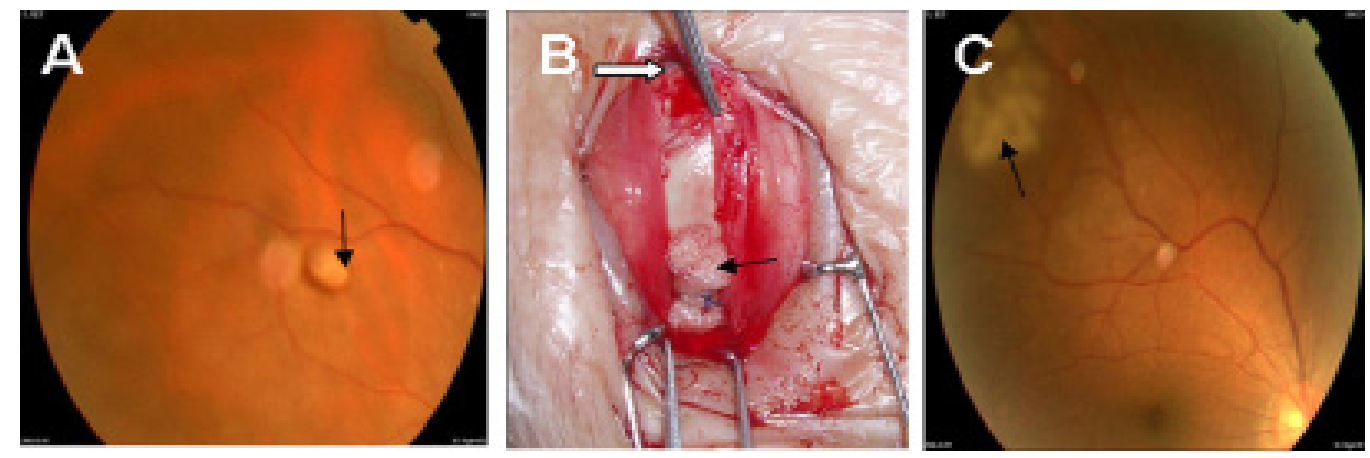

Figure 1. Rhegmatogenous retinal detachment (RRD) with a round hole treated by one " 8 " shaped scleral suture. A. RRD with a round hole and its flap (black arrow) before surgery. B. Buckle of the "8"-shaped scleral suture intraoperatively. The " 8 "-shaped suture (black arrow) holds the radial short silicone sponge on the surface of the sclera behind the equator. There is about $18 \mathrm{~mm}$ from the location of the hole to the limbus (empty arrow). C. Retina reattaches. There are laser spots around the hole after laser photocoagulation and the flap recovers the hole (black arrow). 

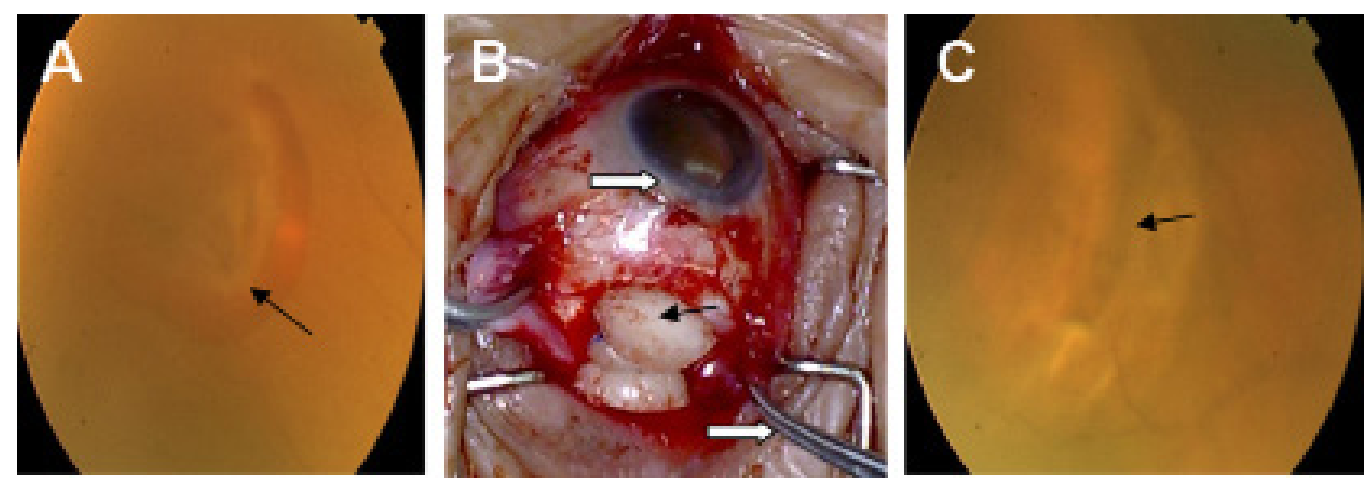

Figure 2. Rhegmatogenous retinal detachment (RRD) with a horse-toe tear treated by one " 8 " shaped scleral suture. A. RRD with a 2DD tear and its flap (black arrow) before surgery. B. The " 8 "-shaped scleral buckle intraoperatively. The "8"-shaped suture (black arrow) holds the radial short silicone sponge on the surface of the sclera behind the equator. There is about $10 \mathrm{~mm}$ from the location of the hole to the limbus (empty arrow). C. Retina reattaches. The tear is located against the scleral crest. The flap of the tear re-closes by the force of the scleral indentation (black arrow).

Then, reassurance of the contraposition of the hole against the scleral crest was accomplished using the IO. If the positions were far from each other or if the hole or tear was not on the crest, the silicone sponge was re-sewn or shifted according to the proper site. If the hole or tear was confirmed to be on the crest, the conjunctive incision was sewed up, and antibiotics solution and dexamethasone were injected beneath the conjunctiva.

\section{Statistical analysis}

For statistical analysis, Snellen visual acuity (VA) measures were converted to a logarithmic scale, i.e. logarithm of the minimum angle of resolution (logMAR). Statistical analysis comparing the means of the preoperative VA and postoperative VA was performed using oneway analysis of variance.

\section{RESULTS}

\section{Patients and preoperative findings}

There were 30 eyes ( 30 cases) with primary RRD that fulfilled the inclusion criteria as outlined above. The age of the patients ranged from 17 to 65 years (mean, $43.1 \pm 8.6$ years). There were 17 male (56.7\%) and 13 female patients (43.3\%) included. A total of 18 patients $(60 \%)$ were operated on the right eye and $12(40 \%)$ on the left eye.

There were 16 horseshoe tears and 14 round or oval holes. All of the tears were located in the superior or superior-temporal quadrants. Six of the holes were located in the inferior-temporal quadrant, and the rest were located on the superior or superior-nasal or temporal quadrants.

\section{Anatomical results}

Of the 30 patients, the retinas of $15(50 \%)$ patients reattached clinically by indirect ophthalmoscope in $24 \mathrm{~h}$, those of 10 (33.3\%) patients reattached in $48 \mathrm{~h}$, and those of 5 
$(16.7 \%)$ patients reattached in one week. All of the breaks or tears lay against the scleral crest. The axis of the buckling sponge was radial in 21 eyes (70\%) and was horizontal or arc-shaped in the others $(30 \%)$. No sub-retinal fluid drainage was needed for any of the patients.

\section{Functional results}

After 6 months of follow-up, the best-corrected visual acuity (BCVA) in 27 eyes increased, and those of the other 3 eyes were unchanged. The mean preoperative VA (in logMAR) was $0.738 \pm 0.368$, and the mean postoperative BCVA (in logMAR) was $0.422 \pm 0.278$, and the difference was statistically significant $(\mathrm{P}<0.05)$.

\section{Complications}

No significant intra-operative complications, such as sub-retinal hemorrhage or incarceration, occurred in any of the 30 eyes. Two weeks after the operation, there was only one patient whose sponge was exposed from the infra-temporal conjunctiva. Because the fundus recovered well, the sponge was taken off immediately to prevent potential infection. The retina maintained its position after 8 months of follow-up.

\section{DISCUSSION}

MSB, originally proposed by Lincoff and Kreissig, has been the preferred external procedure for treating RRDs for at least 40 years; however, it is hard to delineate the exact size of minimal buckling for a specific case. This depends on the case itself and on the routines of the surgeon himself (Lincoff and Kreissig, 1972; Kreissig et al., 1995; Lincoff and Kreissig, 2000; Rezaei and Abrams, 2005; Hanyi et al., 2011; Schimel et al., 2012). In general, retina specialists intentionally expand the range of the buckling to increase the rate of the first operation, and at least two sutures are favored.

On the basis of our previously reported experience with MSB for RRDs (Hanyi et al., 2011), we here applied a refined MSB procedure using one " 8 "-shaped scleral suture to treat primary RRDs.

In this series, a 7-mm wide silicone sponge was fixed with a 6-0 nylon suture in an " 8 " shape, and the distance between the stitch and the edge of the sponge was less than $1 \mathrm{~mm}$ on each side. Theoretically, the maximal diameter of the crest indenting into the vitreous cavity would be at least $7 \mathrm{~mm}$. This range should cover most of the retinal breaks, which are less than 2 DD or $3 \mathrm{~mm}$ in diameter (Laatikainen and Tolppanen, 1985; Coffee et al., 2007; Daling et al., 2008). According to the size or the height of the retina hole or tear, the scleral depth indented or the height of the crest would be modified by the tightness of the suture, the width of the sponge, and the width of the suture. If the size of the hole or tear is large, tighter sutures would be applied to increase the height of the crest, and consequently, the diameter of the crest would increase. Furthermore, if the hole or tear is small, we could also tailor the sponge to be narrower in order to match the size. The length of the sponge can be adjusted according to the length and the axis of the hole or tears, especially for horse-toe tears. In our experience, the length of the sponge was generally less than $10 \mathrm{~mm}$, which was sufficient for sealing the hole or tears. The 30 eyes of recruited patients were buckled by this procedure, and all of the retinas 
reattached after buckling within less than $72 \mathrm{~h}$.

During surgery, the surgical trauma to the eyeball was decreased substantially. First, the incision of the conjunctiva was "T"-shaped, which was limited to only the quadrant with the hole or tear. Second, the rectus muscles were not distracted by the sutures in order to expose the sclera. The corresponding scleral site of the hole or tear was exposed to be wide enough only by rotation of the eyeball by gently stretching the insertion of the muscles with forceps.

With the application of all of these modified and refined procedures, the MSB shows merit in reducing the incidence of surgical injuries, is easier and quicker to perform, and results in more rapid recovery. In some cases, 20 min was sufficient to perform the MSB. In our review, only one scleral suture to hold the sponge against the sclera was the most refined MSB procedure to treat such an RRD, which has not been reported before. It is necessary to expand this kind of modified and refined MSB.

Of course, due to the limited width of the crest by a single "8"-shaped suture, we established strict inclusion criteria, as described above. Biasing this patient sample guaranteed a higher success rate. With additional experience, the indications of a single " 8 " suture will be expanded to some degree.

Although sub-retinal drainage procedure has not been applied for many years, the mechanism of absorption of the subretinal fluid remains controversial (Hammer, 1981; Mester et al., 2002). In two physical models, Clemens et al. (1987) and Foster et al. (2010) suggested that liquid laminar and Bernoulli effects will be formed after the hole or tears are buckled, prompting the sub-retinal fluid to flow back to the vitreous cavity. Furthermore, they indicated that rapid eye movement could help the outflow of the subretinal fluid into the vitreous cavity. It was unnecessary to fix the eyeball or limit the movement of the contra-lateral eye to promote the sub-retinal fluid absorption. In our series, no case was operated with sub-retinal drainage and the sub-retinal fluids in most of the patients were absorbed the following day. The longest duration of sub-retinal fluid was $72 \mathrm{~h}$. The results showed that it was not necessary to drain the fluids below the retina or to block or seal the retinal breaks during the operation. Instead, two conditions were encountered when we performed the traditional surgery, and the intraoperative outcomes were both satisfactory. The first was that although the retina lay against the crest intra-operatively after drainage, in some RRDs, the retina detached post-operatively. The second was that the retina on the plateau of the crest stayed detached while the post-crest retina was attached. However, using this new method, neither of these conditions was encountered. The outcomes showed that the wider range of buckling or indentation did not seal or block the fluid more than that of minimal scleral buckling. Liquid laminar and/or Bernoulli effects were not formed in the former condition; therefore, the fluid could not be pumped out, and even the fluid in the vitreous cavity could flow into the sub-retinal space as before.

Due to the refined procedure of MSB and the omission of sub-retinal drainage of the fluid, which is the standard operation of classical SB, the overall surgery outcome was greatly improved and the surgical time was also shortened substantially. Most of the surgical durations in our series were approximately $30 \mathrm{~min}$, which is markedly less than the surgery times reported previously (Sagong and Chang, 2010). In this sense, this procedure can shorten or change the learning curve of SB.

Since a primary vitrectomy with a $20 \mathrm{G}$ probe was first applied for RRD in 1985, (Escoffery et al., 1985), $25 \mathrm{G}$ or $27 \mathrm{G}$ probes have been employed more recently for treating such RRDs. However, the primary choice, vitrectomy or MSB, remains controversial (Schwartz and Flynn, 2006; Sun et al., 2012). A recent meta-analysis (Sun et al., 2012; Soni et al., 2013) 
found no significant differences in the proportions of primary reattachment and postoperative BCVA after 6 months or more in pseudophakic/aphakic eyes, whereas SB-treated phakic eyes showed better postoperative BCVAs after 6 months or more. In our series, besides the virtues of SB, a refined MSB protocol was established, which features a shorter surgical time, less incision size, and minimal surgical trauma.

In conclusion, the refined one "8"-shaped suture of scleral buckling without sub-retinal drainage was verified to be an efficient procedure for treating selected RRDs. This procedure is an excellent example of "minimally invasive" segmental buckling.

\section{REFERENCES}

Clemens S, Kroll P, Stein E, Wagner W, et al. (1987). Experimental studies on the disappearance of subretinal fluid after episcleral buckling procedures without drainage. Graefes Arch. Clin. Exp. Ophthalmol. 225: 16-18.

Coffee RE, Westfall AC, Davis GH, Mieler WF, et al. (2007). Symptomatic posterior vitreous detachment and the incidence of delayed retinal breaks: case series and meta-analysis. Am. J. Ophthalmol. 144: 409-413.

Daling X, Wen L, Min H and Tong L (2008). Clinical observation of intravitreal dispersion of retinal pigment epithelium cells during external approach microsurgery of retinal detachment. Chin. J. Pract. Ophthalmol. 26.

Escoffery RF, Olk RJ, Grand MG and Boniuk I (1985). Vitrectomy without scleral buckling for primary rhegmatogenous retinal detachment. Am. J. Ophthalmol. 99: 275-281.

Foster WJ, Dowla N, Joshi SY and Nikolaou M (2010). The fluid mechanics of scleral buckling surgery for the repair of retinal detachment. Graefes Arch. Clin. Exp. Ophthalmol. 248: 31-36.

Hammer ME (1981). Retinal re-attachment forces created by absorption of subretinal fluid. Doc. Ophthalmol. Proc. Ser. 125: 61-75.

Hanyi M, Jin M and Fei G (2011). Minimal scleral buckling without subretinal drainage for rhegmatogenous retinal detachment. Med. J. PUMCH 2: 357-361.

Kreissig I, Simader E, Fahle M and Lincoff H (1995). Visual acuity after segmental buckling and non-drainage: a 15-year follow-up. Eur. J. Ophthalmol. 5: 240-246.

Laatikainen L and Tolppanen EM (1985). Characteristics of rhegmatogenous retinal detachment. Acta Ophthalmol. 63: $146-154$.

Lincoff H and Kreissig I (1972). The treatment of retinal detachment without drainage of subretinal fluid. (Modifications of the Custodis procedure. VI). Trans. Am. Acad. Ophthalmol. Otolaryngol. 76: 1121-1133.

Lincoff H and Kreissig I (2000). Changing patterns in the surgery for retinal detachment: 1929 to 2000. Klin Monbl Augenheilkd 216: 352-359.

Mester U, Anterist N, Kroll P and Brieden-Azvedo S (2002). The role of the vitreous in retinal detachment surgery with external buckling. Ophthalmologica 216: 242-245.

Rezaei KA and Abrams GW (2005). The History of Retinal Detachment Surgery. Kreissig I. Primary Retinal Detachment (option for repair). Springer, New York.

Rumpf J (1976). Jules Gonin. Inventor of the surgical treatment for retinal detachment. Surv. Ophthalmol. 21: 276-284.

Sagong M and Chang W (2010). Learning curve of the scleral buckling operation: lessons from the first 97 cases. Ophthalmologica 224: 22-29.

Schepens CL (1951). Progress in detachment surgery. Trans. Am. Acad. Ophthalmol. Otolaryngol. 55: 607-615.

Schepens CL, Okamura ID and Brockhurst RJ (1957). The scleral buckling procedures. I. Surgical techniques and management. AMA Arch. Ophthalmol. 58: 797-811.

Schimel AM, Grand MG and Flynn HW, Jr. (2012). Surgery for retinal detachment: does experience matter? Retina 32: $1-3$.

Schwartz SG and Flynn HW (2006). Primary retinal detachment: scleral buckle or pars plana vitrectomy? Curr. Opin. Ophthalmol. 17: 245-250.

Soni C, Hainsworth DP and Almony A (2013). Surgical management of rhegmatogenous retinal detachment: a metaanalysis of randomized controlled trials. Ophthalmology 120: 1440-1447.

Sun Q, Sun T, Xu Y, Yang XL, et al. (2012). Primary vitrectomy versus scleral buckling for the treatment of rhegmatogenous retinal detachment: a meta-analysis of randomized controlled clinical trials. Curr. Eye Res. 37: 492-499. 